\title{
Le bestiaire au dix-neuvième siècle \\ Animalité, pauvreté et criminalité dans Les mystères de Paris
}

Nigel LEZAMA, Université Brock

La restructuration du domaine épistémologique qui a lieu à la fin du dix-huitième siècle et qui instaure la prédilection positiviste, entraîne avec elle un besoin de classer le monde naturel (voir Foucault 1966) et impose ainsi un regard sur l'animal. Celui-ci aura à gagner et à perdre dans le nouveau système classificateur dans lequel il se trouve. À gagner : sur le plan juridique, les droits de l'animal et sa protection de la souffrance deviennent monnaie courante dans le discours public ; en 1802, l'Institut national de France lance son concours annuel du meilleur mémoire qui réfléchit à la question «Jusqu’à quel point les traitements barbares exercés sur les animaux intéressent-ils la morale publique. Et, conviendrait-il de faire des lois à cet égard ? ${ }^{1}$. Cette prise de conscience des droits de l'animal atteindra son apogée, non pas sans certains reculs, en 1850 avec l'entérinement de la Loi Grammont sur le mauvais traitement des animaux

domestiques. À perdre : selon la pensée matérialiste, héritée des Lumières, l'animal est toujours conçu comme une «machine animée $»^{2}$; encore en 1802, dans son traité Rapports du physique et du moral de l'homme, le médecin-philosophe P.-J.-G. Cabanis élabore une théorie éthique fondée sur l'analogie du fonctionnement du corps avec celui de la machine.

Or, la supériorité de l'organisation des nerfs et du cerveau dans l'homme, et l'empire qu'ils acquièrent journellement par l'exercice même de leurs plus nobles facultés, ou par la production des idées et des sentiments, font que chez lui la vie semble tenir, moins que chez tout autre animal, à l'état mécanique et matériel des organes ; que chez lui, on peut observer, plus distinctement que chez tout autre, les empreintes fixes ou variables de ce moule interne, auquel se rapportent toutes les formes et tous les actes extérieurs. (499-500)

L'homme, comme l'animal, est marqué par un fonctionnement mécanique (l'esprit n'est qu'une fonction mécanique parmi d'autres, selon Cabanis) ; ce n'est que grâce à une organisation intérieure et organique supérieure à celle de l'animal que l'homme est moins gouverné par son corps et ses organes.

De son côté, le discours économique du dix-neuvième siècle occulte le «travail» de l'animal ; dans Le capital (1867), par exemple, Karl Marx affirme que le travail est le propre de 
l'homme parce qu'il agit sur la nature et arrive à la modifier grâce à la conscience qu'il a de son ouvrage. Il propose :

Notre point de départ c'est le travail sous une forme qui appartient exclusivement à l'homme. Une araignée fait des opérations qui ressemblent à celle du tisserand, et l'abeille confond par la structure de ses cellules de cire l'habileté de plus d'un architecte. Mais ce qui distingue dès l'abord le plus mauvais architecte de l'abeille la plus experte, c'est qu'il a construit la cellule dans sa tête avant de la construire dans sa ruche. Le résultat auquel le travail aboutit préexiste idéalement dans l'imagination du travailleur. (728)

L'animal, à l'encontre de l'homme, agit par instinct, voire naturellement ; selon Marx, l'abeille, dont la production de cellules dans la ruche se rapproche du travail de l'architecte, n'a aucune conscience préalable du résultat de son ouvrage, ce qui empêche de considérer ce résultat comme « travail » en tant que tel. De surcroît, l'animal agit directement sur les matières naturelles, tandis que l'homme, même le plus primitif, conçoit des instruments de travail avec lesquels il opère sa transformation de la nature. «Le moyen de travail est une chose ou un ensemble de choses que l'homme interpose entre lui et l'objet de son travail comme conducteurs de son action » (729). Selon Marx, seul le travail conscient et médiatisé de l'homme a une valeur intrinsèque. L'animal reste dans l'angle mort de sa pensée.

L'animal s'impose toutefois à la littérature. Honoré de Balzac mesure l'homme à l'aune de l'animal : l'«Avant-propos » de La comédie humaine dévoile la source d'inspiration de son auteur : «Pénétré de ce système bien avant les débats auxquels il a donné lieu, je vis que, sous ce rapport, la Société ressemblait à la Nature. La Société ne fait-elle pas de l'homme, suivant les milieux où son action se déploie, autant d'hommes différents qu'il y a de variétés en zoologie ? » (9). Le personnage balzacien est formé par son milieu tout comme, selon le discours scientifique, l'animal est marqué par le monde naturel qu'il habite ; ainsi, le travail proprement scientifique de l'œuvre balzacienne sera d'identifier les règles qui gouvernent le monde des hommes, tout comme celui du naturaliste sera de relever celles du monde animal. La figure animale joue également un rôle dans le monde hugolien ; dans Les misérables, en décrivant l'inspecteur Javert, le narrateur exprime la croyance que dans chaque homme existe un animal, visible si l'âme de l'homme était observable.

Dans notre conviction, si les âmes étaient visibles aux yeux, on verrait distinctement cette chose étrange que chacun des individus de l'espèce humaine correspond à quelqu'une des espèces de la création animale ; et l'on pourrait reconnaître aisément cette vérité à peine entrevue par le penseur, que, depuis 
l'huître jusqu'à l'aigle, depuis le porc jusqu'au tigre, tous les animaux sont dans l'homme et que chacun d'eux est dans un homme. (212)

Pour Victor Hugo, il n'existe aucune scission entre l'homme et l'animal ; l'humain est marqué par un for intérieur foncièrement animal. Fortement influencé par la tradition physiognomonique, Hugo pousse plus loin le système classificateur de Balzac dans la mesure où il efface toute distinction entre homme et animal : il s'agit d'une première manifestation de la bête humaine. L'auteur des Misérables lit, dans la ressemblance aux bêtes, des traits de caractère chez l'homme. Chez Charles Baudelaire, la vision allégorique, élaborée dans son œuvre poétique, montre la chute de l'art et de l'artiste dans le système économique moderne grâce à ses mises en scène d'animaux, tels l'albatros ou le chien. Dans « Le chien et le flacon », par exemple, le poète met au clair l'analogie du chien et du public : «-Ah ! misérable chien, si je vous avais offert un paquet d'excréments, vous l'auriez flairé avec délices, et peut être dévoré. Ainsi, vous-même, indigne compagnon de ma triste vie, vous ressemblez au public, à qui il ne faut jamais présenter des parfums délicats qui l'exaspèrent, mais des ordures soigneusement choisies » (284). Dans le discours littéraire, tout comme le scientifique, le philosophique et l'économique, force est de constater que l'homme se mesure face à l'animal.

\section{Pauvreté et animalité}

Au moment de la mise en discours de l'animal et de son statut ontologique et politique au dix-neuvième siècle, la recrudescence de la pauvreté fait également marcher la machine discursive. La perception d'une crise de misère ouvrière fait du pauvre une fixation anxiogène que de nombreux écrivains et penseurs sociaux de l'époque, cherchant à protéger un ordre social en déstabilisation continue, essayent de corriger. Pour ce qui est de la construction discursive du pauvre, la bête a également son fardeau à porter. Dans les nombreux textes ethnographiques qui voient le jour pendant la première moitié du siècle, le pauvre est construit par un appareil descriptif axé sur une animalité ignoble qui le distancie de ses homologues des classes aisées.

Malgré une bonne volonté et un vif désir de trouver les moyens de guérir cette «plaie sociale », Eugène Buret, par exemple, creuse le problème de la pauvreté dans son ouvrage De la misère des classes laborieuses en Angleterre et en France $(1840)^{3}$, en construisant l'abjection du pauvre par le biais de métaphores animalières : «L'homme ne se multiplie pas à la façon des insectes immondes ; s'il succombe sous le poids d'une fécondité impudente, c'est que l'homme a 
disparu et que l'animal seul a survécu aux attaques prolongées de la misère » (236), décrie l'ethnographe. Dans la pensée sociale, la misère subie pendant une longue période réduit l'homme à un état de sauvagerie avoisinant l'état animal. Il est évident que, sur le continuum anthropologique, la bête occupe un des pôles et l'homo ideologicus en occupe l'autre ; l'angoisse évidente dans la pensée sociale provient de la croyance que l'animal reste fixe à son extrême du continuum tandis que l'homme a le malheur de pouvoir quitter son statut dominant pour s'approcher plus ou moins de l'abjection animale.

Les mystères de Paris, publié en feuilleton dans le Journal des Débats entre juin 1842 et octobre 1843, se révèle particulièrement propice pour l'analyse du discours portant sur les enjeux sociaux, en général, et sur la question animale, en particulier, en tant que texte à l'intersection du littéraire et du sociopolitique ${ }^{4}$. Dans cette œuvre, le feuilletoniste Eugène Sue manipule plusieurs préoccupations sociales afin d'alimenter la trame narrative et de tenir son lectorat en haleine tout au long de la publication. Le roman-feuilleton, en tant que produit littéraire à grand tirage, devait interpeler son public grâce à un tissu diégétique formé de multiples fils discursifs provenant du réel. Dans cette optique, la raison pour laquelle le feuilletoniste peuple son monde de pauvres et de criminels est évidente: la misère est la question sociale la plus poignante de l'époque 5 . L'animal a aussi son rôle à jouer dans la mise en scène et l'élaboration du personnage marginal ; pourtant, à l'encontre de la production littéraire citée plus haut, dans Les mystères de Paris, la figure animale demeure floue sur le plan discursif : l'animal n'est pas seulement l'aune avec laquelle mesurer le personnage ; il n'est pas non plus simplement une formule abrégée pour pointer l'excentricité. Afin d'élaborer la sémantique animale chez Sue, la suite de cette discussion privilégiera la représentation de deux figures marginales des Mystères de Paris afin de donner sens à l'usage de la figure animale dans le monde suélien. Je m'intéresserai d'abord au Maître d'école, le criminel le plus redoutable du monde suélien. Ensuite, je contrasterai la façon dont Sue représente celui-ci avec une autre figure marginale, le Chourineur, un ancien bagnard qui arrive à réintégrer l'ordre social. Grâce à cette analyse de l'animal comme figure rhétorique dans la construction des personnages marginaux, il deviendra évident que la représentation animale dans le feuilleton de Sue reste équivoque en tant que baromètre social et reflète ainsi le statut ambivalent qu'occupent à la fois le pauvre et l'animal au dix-neuvième siècle. 


\section{Criminalité animale}

Dans le monde suélien, le statut social du personnage excentrique se lit à fleur de peau. Le médium du feuilleton et son moyen de production privilégient un style littéraire cousu de fil blanc ; dans la peinture de certains personnages, l'animalité est mise en avant afin de souligner le non-conformisme idéologique. Ainsi, lorsque le narrateur pose son regard sur le Maître d'École, le chef du monde criminel, l'animalité de son corps est mise au premier plan :

Sa figure était sillonnée en tous sens de cicatrices profondes, livides ; l'action corrosive du vitriol avait boursoufflé ses lèvres ; les cartilages du nez ayant été coupés, deux trous difformes remplaçaient les narines. Ses yeux gris, très clairs, très petits, très ronds, étincelaient de férocité ; son front, aplati comme celui d'un tigre, disparaissait à demi sous une casquette de fourrure à longs poils fauves... on eut dit la crinière d'un monstre. (70)

La déshumanisation va de pair avec l'animalisation, signe qui pointe le statut excentrique du personnage, à l'instar de la représentation des ouvriers indigents dans le texte de Buret. L'écrivain se sert de métaphores animalières pour signaler le statut inférieur de l'individu représenté. Pour Buret, la misère réduit l'homme à un état bestial ; le pauvre perd les facultés essentielles à son humanité. Chez Sue, il y a un processus analogue: l'animalité signale également un personnage hors-la-loi, une source de déstabilisation de l'ordre social. Sue amplifie le statut « animal » du brigand afin de souligner son écart du centre idéologique et de connoter le danger que le personnage pose à la société civile ; dans le cas du Maître d'école, l'animalité n'est pas inoffensive, comme l'image de l'insecte dont se sert Buret pour décrire l'ouvrier misérable. Il s'agit, en l'occurrence, d'une animalité dont la force et la férocité signifient un risque au bon fonctionnement de la société.

Le texte souligne en plus que le criminel s'est lui-même défiguré après s'être évadé du bagne. L'humanité consciemment gommée est tout à l'encontre de l'état du pauvre - abruti malgré lui par un travail insuffisamment rémunéré - et annonce un personnage rendu néfaste de son plein gré. Férocité du regard, front aplati du carnivore, même le vêtement - la casquette à poils - surdétermine sa non-appartenance à la race d'homme. Dans la suite de la description, la peinture du corps de l'ancien bagnard ne réfute rien au zoomorphisme de sa physionomie ni de son vêtement.

[II] n'avait guère plus de cinq pieds deux ou trois pouces ; sa tête, démesurément grosse, était enfoncée entre ses deux épaules larges, élevées, puissantes, charnues, qui se dessinaient même sous les plis flottants de sa blouse de toile écrue ; il avait 
les bras longs, musculeux ; les mains courtes, grosses et velues jusqu'à l'extrémité des doigts ; ses jambes étaient un peu arquées, mais leurs mollets énormes annonçaient une force athlétique. (70)

Corps ramassé, bras longs et poilus, jambes arquées et musclées : s'il n'était pas un personnage d'un feuilleton du dix-neuvième siècle, le Maître d'École pourrait facilement s'intégrer au plateau de tournage du film La planète des singes ${ }^{6}$.

Dès l'ouverture du siècle, le domaine scientifique se prépare pour l'évolutionnisme de Darwin. Bien que l'intérêt scientifique porté à nos cousins les singes voie le jour dès le milieu du dix-huitième siècle ${ }^{7}$, ce sont les expéditions maritimes, les spécimens vivants rapportés et le questionnement sur l'origine de l'orang-outang, vers la fin de ce siècle qui imposent une mise en cause de l'évidence et de la pérennité de la croyance en l'exception humaine (Serna) ${ }^{8}$. Dans son Histoire naturelle des singes et des makis, publiée en 1797, Jean Baptiste Audebert trahit l'angoisse ressentie face au singe, ce représentant d'une «humanité dégradée » :

[...] l'homme, étonné à l'aspect inattendu de ces animaux, et pour ainsi dire, honteux de reconnaître le plus grand nombre de ses rapports dans un être qui offre des traits de l'humanité dégradée, supposa aux singes une nature et un entendement supérieur au reste des bêtes. De là ces histoires merveilleuses, ces rêveries, fruits d'une imagination mensongère que ses prédécesseurs ont accumulés en parlant de ces animaux. (Audebert, cité dans Serna 250)

Le naturaliste prête voix, peut-être malgré lui, au malaise de voir un animal qui, grâce à un isomorphisme, semble enjamber le gouffre le séparant de l'homme. Se voir singer par cette espèce indigne, c'est voir ce qui est indigne dans sa propre espèce. Effectivement, le singe met un bâton dans la roue de ce que Giorgio Agamben dénomme la «machine anthropologique » dans L'ouvert. De l'homme à l'animal, son exégèse du système ontologique qui perpétue la distinction discursive de l'homme et de l'animal.

Dans la mise en scène du Maître d'école, Sue laisse transparaître un malaise similaire à celui évident dans le discours d'Audebert. Tout comme le singe, ce criminel illustre le type de l'humanité dégradée évoquée par le naturaliste. Malgré la distance séparant le criminel de son cousin plus honorable, homo ideologicus, ce sont les similarités qui ébranlent les convictions. Dans une scène qui suit l'introduction de ce chef des criminels, le Maître d'école et sa compagne, également abjecte et - de façon pertinente - nommée la Chouette, rencontrent le héros-flâneur du roman, le prince Rodolphe, dans la rue. Le narrateur évoque l'inquiétante 
étrangeté ${ }^{9}$ provenant de l'apparente intégration de ce couple excentrique et dégénéré dans le monde des hommes :

Ces deux personnages étaient complètement métamorphosés : le brigand avait abandonné ses méchants habits et son air de brutalité féroce ; il portait une longue redingote de castorine verte et un chapeau rond ; sa cravate et sa chemise étaient d'une extrême blancheur. Sans l'épouvantable hideur de ses traits et le fauve éclat de son regard, toujours ardent et mobile, on eût pris cet homme, à sa démarche paisible, assurée, pour un honnête bourgeois. (Sue 122)

L'animalité du criminel - évoquée par les termes «brutalité féroce » et «fauve éclat de son regard »-s'éclipse presque derrière le vêtement bourgeois. Cette mise en scène rend évidente l'angoisse suscitée par la porosité de la frontière séparant le centre de la marge tout autant que celle qui sépare l'homme de l'animal.

Dans son livre Nudité et pudeur. Le mythe du processus de civilisation, Hans Peter Duerr rappelle que le vêtement est l'outil essentiel au passage de la « sauvagerie » à la « civilisation » dans la société européenne : «les Européens, surtout depuis le seizième siècle, auraient entrepris de progressivement 'refouler tout ce qu'ils ressentent en eux-mêmes comme relevant de leur 'nature animale',. Ils auraient donc amorcé ce que les moralistes écossais des Lumières ont appelé le passage de la 'savage' à la 'civil society', ou encore 'from rude to refined' » (1-2). Que cette évolution de la sauvagerie à la civilité ait été si facilement déjouée par le criminel-animal fut une source d'angoisse pour une société obsédée par la dégénération de l'espèce, ce qui est évident dans la fixation sociale sur le cas des excentriques et des marginalisés. L' «évolution » vestimentaire du Maître d'école et de sa conjointe en figures doxologiques signale que le processus inverse, la dégénérescence de l'individu, pourrait avoir lieu aussi facilement que l'intégration sournoise des marginaux vers le centre.

La mise en scène de l'animalité chez Sue est particulière dans la mesure où elle reste, en grande partie, axée sur des éléments antisociaux et une représentation manichéenne. L'humanité s'exhale du bon et la férocité animale émane du méchant. L'historien Pierre Serna, travaillant récemment sur la dialectique homme-animal dans son article «Droits d'humanité, droits d'animalité à la fin du dix-huitième siècle, ou la matrice du 'racisme social' en controverse », relève entre les lignes du discours du naturaliste Salaville un plaidoyer surprenant contre l'esclavage ${ }^{10}$. Une autre découverte de Serna retiendra pourtant mon attention : la théorie du végétarisme qu'il identifie dans un autre texte, cette fois-ci de Delamétherie. Dans son traité $D e$ 
l'homme considéré moralement, de ses mours, et celles des animaux (1802), le naturaliste matérialiste Delamétherie propose l'existence de deux catégories de vivants: les hommes «sociaux » d'un côté, et les animaux et les hommes «de nature» de l'autre ; le végétarisme primordial de l'homme est le critère distinguant les deux catégories. Selon le naturaliste, à un moment reculé de l'histoire, l'homme a abandonné une pratique exclusivement frugivore, ce qui a mené à sa chute: «Cette époque transforma les mœurs des humains qui ont, à partir de ce moment, égorgé sans scrupules les autres animaux. La violence de sang froid, la guerre, provoquèrent cette altération qui, en leur conférant toujours plus d'intelligence, les plongea dans l'agression permanente » (Serna 253). C'est l'appétit carnassier qui est à la source de la violence humaine. L'animalité criminelle dans le monde suélien privilégie la férocité, la violence et l'agression qui renvoient toutes vers la pratique des animaux carnivores. Grâce à sa mise en scène de l'animalité criminelle, il est clair que Sue se met au diapason de ce discours scientifique voyant dans la férocité de l'antisocial l'héritage du goût sanguinaire développé par l'homme primordial suite à sa «chute» dans le carnivorisme. Ainsi, la violence criminelle s'affirme comme une dégénération de l'homme éthique.

\section{Dompter l'animal}

Le basculement vers l'antisocial, figuré par l'animalité surdéterminée du criminel, est un aspect de la sémantique animale dans le monde suélien. La trajectoire diégétique d'un autre criminel redouté, le Chourineur, montre que la bête humaine peut être domptée et réhabilitée au nom de l'idéologie. Le Chourineur, ainsi nommé pour sa prédilection pour l'arme blanche, est le premier personnage du feuilleton présenté au lecteur. Malgré son statut criminel et ses premières mauvaises actions dans la diégèse, son animalité ne se présente pas en lien à sa criminalité, tel le Maître d'école ; ce n'est qu'une fois «dompté » que le Chourineur s'animalise. Dans le premier chapitre, le narrateur entrevoit « un homme d'une taille athlétique, vêtu d'une mauvaise blouse » (32) se dirigeant vers la Cité par une nuit pluvieuse. L'entrée du Chourineur dans la diégèse n'est pas marquée par son animalité excentrique ; le narrateur souligne qu'il s'agit bel et bien d'un homme, remarquable plutôt pour sa taille et sa force.

Le Chourineur est néanmoins un tyran dans la pègre ; après avoir été vaincu dans une échauffourée avec le prince Rodolphe, le narrateur évoque plus clairement le brigand : il s'agit d'un «homme de haute taille et de constitution athlétique, [il] a des cheveux d'un blond pâle 
tirant sur le blanc, des sourcils épais et d'énormes favoris d'un roux ardent » (39). Cette peinture est tout à l'encontre de celle du Maître d'école : l' «humanité » du Chourineur n'est pas éclipsée par un corps excessivement animal. Le narrateur le situe pleinement du côté humain dans l'équation anthropologique : «Malgré son terrible surnom, les traits de cet homme expriment plutôt une sorte d'audace brutale que la férocité ; quoique la partie postérieure de son crâne, singulièrement développée, annonce la prédominance des appétits meurtriers et charnels » (39). Ce personnage se trouve à l'extérieur du centre idéologique, mais son statut excentrique n'est point surdéterminé en peignant un corps animalisé ${ }^{11}$. La détermination que le Chourineur n'est pas féroce, mais plutôt audacieux, est le moyen grâce auquel le texte prépare sa réintégration à l'ordre social à venir. À l'encontre de la férocité (du Maître d'école, par exemple), l'audace du Chourineur est un trait qui a le potentiel d'être engagé dans l'appui de la doxa.

En l'occurrence, il est pertinent que l'animalité du Chourineur ne soit pas non plus une animalité inquiétante, mais plutôt une animalité qui peut être domptée et mise au service de la société bourgeoise. La raclée que subit le brigand aux mains du prince Rodolphe a l'effet de le réintégrer au sein de l'idéologie : dès qu'il se dit vaincu, le désordre potentiel est effectivement désamorcé ; le meurtrier redoutable devient le chien de garde de son vainqueur, ce qu'il avoue au prince : «Que le diable me brûle si je sais pourquoi je me sens pour vous comme qui dirait l'attachement d'un bouledogue pour son maître ; mais c'est égal, ça est. C'est plus fort que moi, je ne m'en brûle plus... ça vous regarde, arrangez-vous » (91). L'animalité, qui ébranlait l'anthropocentrisme par une rencontre de l'altérité abjecte avec une familiarité angoissante, comme celle du Maître d'école, étaye désormais l'ordre social. L'animalité de celui-ci se caractérise par la férocité et la sauvagerie ; il s'agit d'une animalité qui renonce à l'humain, tandis que celle du Chourineur, choisie de son plein gré comme le Maître d'école, se singularise par sa sujétion à l'humain. Ce qui était la marque d'une altérité radicale et inquiétante est désamorcé en le plaçant au service de l'ordre établi. De surcroît, cette représentation d'une animalité domptée appuie l'ordre social pour une autre raison : le nouveau rôle qu'assume le Chourineur redouble la structure idéologique d'une société cherchant à occuper les pauvres par un travail assujettissant au profit des classes dominantes. C'est la raison pour laquelle Marx s'en prend à Sue dans sa critique littéraire du feuilleton, La sainte famille, pour cette réduction de statut de l'homme au bouledogue : «L'ancien boucher est métamorphosé en chien. À partir de ce moment, toutes ses vertus se résoudront en la vertu du chien, le pur 'dévouement' à son maître » 
(169). Selon Marx, la réduction idéologique à l'état de bête est un sort qui n'attend que le pauvre, l'ouvrier indigent, tous ceux qui vivent à l'encontre de l'ordre social. La critique de Marx repose sur le fait que Sue efface l'autonomie du Chourineur, le propre de l'homme libre, pour la remplacer par une animalité soumise et tout aussi abjecte que l'animalité dangereuse du Maître d'école. Transformé en bouledogue, le Chourineur ne gagne rien en dignité humaine, mais en devient le serviteur ; il ne bénéficie d'aucune amélioration réelle de statut.

Vu la conception marxiste du travail animal, il y a lieu de revenir sur sa lecture de la transformation en animal du Chourineur. Bien qu'il n'ait pas tort d'y lire l'aliénation du meurtrier par l'auto-identification au chien que Sue impose à ce personnage, à la lumière de la conscience grandissante de l'animal et de son rôle dans la société au dix-neuvième siècle, l'animalité idéologique pourrait se lire comme un moyen de rapprocher l'animal et l'humain et, ainsi, de montrer la volonté d'améliorer la situation de chacun. Le Chourineur gomme son humanité en se disant «le bouledogue » du prince, mais il retrouve néanmoins un rôle social dans le centre et, ainsi, quitte la marge. Grâce à son identification, il est possible de déterminer que le Chourineur trouve sa raison d'être et une identité dont il est l'auteur. Le texte semble ainsi valoriser un rôle pour l'animal dans le bon fonctionnement de l'ordre social.

\section{Souffrance animale}

En l'occurrence, la figure animale permet une autre entrée dans le discours sociopolitique à l'œuvre dans le feuilleton : par le biais de la mise en scène du Chourineur, Sue introduit un discours contre la souffrance animale. À la sollicitation de Rodolphe, le Chourineur lui raconte sa vie: enfant vagabond, son premier emploi était comme équarrisseur de chevaux à Montfaucon, un lieu immonde connu depuis le Moyen Âge comme lieu d'exécution publique. Sue s'arrête longuement sur l'épisode de la jeunesse du Chourineur pour mettre en rapport l'abattage et le meurtre ; l'ancien bagnard révèle : "Quand j'ai commencé à chouriner ces pauvres vieilles bêtes, ça me faisait une espèce d'effet : au bout d'un mois, je n'y pensais plus ; au contraire, je prenais goût à mon état. Il n'y avait personne pour avoir des couteaux affilés et aiguisés comme les miens... » (61). Sue insiste sur l'aguerrissement à la violence par le biais de l'animal. Dans cette peinture, l'agression antisociale de l'ancien bagnard se révèle une qualité acquise par l'abattage de chevaux. Au début de son emploi, le Chourineur est décontenancé par cette violence sanglante, malgré l'appétit qui s'annonce, selon le narrateur, de façon 
phrénologique. La mise en scène du texte soutient que la violence n'est pas innée, mais s'apprend et s'accroît : de la violence envers l'animal à la violence entre hommes, il n'y a qu'un pas à faire. Or, ce tableau se lit comme un discours contre la violence animale et fait écho à la pensée végétaliste de Delamétherie à l'orée du siècle.

Après avoir écouté l'histoire du Chourineur, le prince Rodolphe lui offre une maison et une boucherie afin de récompenser l'humanité inhérente de l'ancien brigand et de l'intégrer à la vie et à l'idéologie bourgeoises en tant que maître-boucher ${ }^{12}$. Il accepte le don, d'abord malaisément, mais ensuite avec gratitude. Afin de remercier le prince, il lui abat une brebis dans une scène qui étaye le discours contre la violence que subit l'animal aux mains de l'homme ; Sue y souligne le lien entre la violence contre l'animal et la dégénération morale. Dans un premier temps, le Chourineur s'adonne avec délectation à sa tâche sanguinaire : il subit ce qui ressemble à une régression évolutionnaire à la seule promesse de l'égorgement. «Et les yeux brillant d'un éclat sauvage, ne s'apercevant plus de la présence de Rodolphe, il souleva la brebis sans effort, et d'un bond il l'emporta dans la tuerie avec une joie féroce »(181). L'emploi des vocables «sauvage » et «féroce » renforce la perception de la dégénération en animalité inhérente à la pratique de l'abattage : c'est l'idée de la saignée qui déclenche l'involution de l'ancien tueur. Après avoir égorgé la bête, qui

poussa un petit bêlement doux, plaintif, tourna son regard mourant vers le Chourineur, $[\ldots]$ deux jets de sang frappèrent le tueur au visage. Ce cri, ce regard, ce sang dont il dégouttait causèrent une épouvantable impression à cet homme. Son couteau lui tomba des mains, sa figure devint livide, contractée, effrayante sous le sang qui la couvrait; ses yeux s'arrondirent, ses cheveux se hérissèrent ; puis, reculant tout à coup avec horreur, il s'écria d'une voix étouffée : - Oh ! le sergent ! le sergent ! (181)

La scène met en opposition l'innocence de l'animal avec la cruauté innée de l'homme. La brebis, animal-signe de l'innocence, inspire toute la compassion dans ce tableau, à l'encontre du Chourineur, dont la représentation conjure les angoisses sociales manipulées par l'idéologie au sujet de la pauvreté et de la criminalité. Face à l'innocence de la brebis, le narrateur appelle le boucher un «tueur» afin de rappeler son statut excentrique et sa nature meurtrière et de souligner l'horreur de l'abattage. Égorgeant la bête, le Chourineur est transporté à son premier meurtre. Une fois revenu à lui, il s'explique devant son bienfaiteur :

quand j'ai entendu le cri de cette pauvre bête qui ne se défendait pas... quand j'ai senti son sang me sauter à la figure... un sang chaud... qui avait l'air d'être en 
vie... Oh ! vous ne savez pas ce que c'est... alors, j'ai revu mon rêve... le sergent... et ces pauvres jeunes soldats que je chourinais... qui ne se défendaient pas.... et qui en mourant me regardaient d'un air si doux... si doux...qu'ils avaient l'air de me plaindre ! (182)

Le spectacle de l'effondrement de l'ancien équarisseur et l'éclaircissement qu'il en donne, touchent Rodolphe, qui comprend désormais que « chez le Chourineur, la bête sauvage, l'instinct sanguinaire avait vaincu l'homme ; mais le remords avait vaincu l'instinct » (182). Dans le for intérieur de l'homme résident deux «natures »: c'est le propre de l'homme de combattre la nature animale pour laisser prévaloir l'humaine. Rodolphe avait préparé une autre récompense pour l'ancien meurtrier, au cas où la boucherie ne lui aurait pas convenu. Cette deuxième option intègre mieux ces premiers mouvements du siècle au nom de la protection des animaux : le prince propose à son donataire une métairie en Algérie où il puisse élever, et non pas abattre, des bestiaux. Le Chourineur part de suite pour Alger. Les personnages et le lecteur s'alignent tous sur la réalisation qu'il existe une équivalence, ou au moins une relation de degrés, entre la violence contre l'animal et celle contre l'homme. La scène d'abattage ouvre une brèche pour le feuilleton dans la discussion idéologique contre la violence animale. La relation étroite que pose le texte entre la souffrance animale et la souffrance humaine devient un argument pour la protection de l'animal.

Sue n'explicite pas un discours contre la souffrance animale, comme ce sera sa tendance plus loin dans le feuilleton avec d'autres enjeux sociopolitiques, tels l'isolement pénitentiaire ou la misère ouvrière, mais son lecteur aurait pu dégager l'idée de base que le basculement de la violence envers l'animal à celle entre hommes se fait facilement : ainsi, protéger l'animal, c'est protéger l'homme. Les débats parlementaires sur la Loi Grammont seront entamés en 1850, soit sept ans après la publication du feuilleton. Selon l'historien Éric Baratay, dans son article « La Souffrance animale, face masquée de la protection aux dix-neuvième et vingtième siècles », l'intérêt pour l'animal lui-même et sa souffrance est évident dans les divers surgissements de conscience politique devant la situation de l'animal. À la lumière de son interrogation de l'enjeu, Baratay suggère que le lien entre la politique animale et le réformisme du dix-neuvième siècle est toujours à développer. La façon dont Sue représente les dominés en mettant l'animalité au premier plan démontre à la fois le statut équivoque de l'animal et l'ambivalence avec laquelle les pauvres sont appréhendés par l'idéologie. 


\section{Conclusion}

Dans le monde suélien, le côté dangereux et antisocial de l'homme est évoqué grâce à une imagerie animale ; cependant, le feuilletoniste se sert également de l'animal pour avancer une pratique plus bienveillante pour l'homme face à l'animal ainsi que pour l'homme face à son homologue. Il est parlant que, dans la mise en scène du Maître d'école, Sue a inventé un personnage qui a gommé son humanité de son propre gré. Dans cette optique, il est évident que la dégénération est un choix et non pas une chute contre laquelle l'individu n'a aucun pouvoir. Bien que l'on puisse imaginer que le Maître d'école suit son libre arbitre - il se défigure, il endosse le costume du bourgeois, comme et quand il veut -, la mise en scène du texte souligne que cette «agentivité » n'est que le choix d'avoir laissé la bête sauvage vaincre l'homme. L'homme-singe est l'externalisation du for intérieur du Maître d'école. Quant au Chourineur, Sue va à contre-courant de la pensée phrénologique : la représentation du Chourineur dément la croyance en une criminalité innée, lisible au niveau du corps de l'individu. La peinture de la jeunesse de cet ancien meurtrier souligne en plus qu'il n'est pas né sanguinaire, mais a appris ce comportement à force de prendre part à la tuerie animale.

En guise de conclusion, force est de constater que ni l'animal ni l'excentrique n'est uniformément surdéterminé dans le monde suélien. La mise en scène du Maître d'École puise des deux mains dans une imagerie zoologique qui souligne l'altérité inquiétante de l'animal tout autant que du criminel. Dans cette optique, Sue s'aligne sur la pensée sur l'animal du début du siècle, telle qu'elle se présente dans le travail du naturaliste Audebert ainsi que dans le penchant matérialiste de certains philosophes et scientifiques du siècle qui restreignent la capacité éthique au fonctionnement mécanique du corps. Cependant, la représentation du Chourineur s'avère plus nuancée. Bien que la mise en scène de l'animalité de l'ancien meurtrier renforce l'image de la dégénérescence du pauvre devant les classes dominantes, l'animal, tout autant que l'excentrique, gagne en statut puisque Sue met en avant le lien attachant l'animal et l'homme. Le feuilletoniste introduit même un nouvel argument contre la violence animale en évoquant le basculement de la violence contre l'animal à la violence entre hommes. Dans cette optique, Les mystères de Paris fraie une voie non seulement vers la question sociale, mais pose un lien étroit entre la violence animale et le désordre social. La scène du Chourineur et de la brebis rend évidente la perspective de Sue quant à la souffrance de l'animal : l'abattage équivaut et mène au meurtre. Ainsi, tout en 
évoquant le monde et les mœurs des marginaux, le feuilleton montre que la question sociale ne peut pas se résoudre sans réfléchir au statut de l'animal.

\section{Bibliographie}

Agamben, Giorgio. L'ouvert. De l'homme à l'animal. Tr. Joël Gayraud. Paris : Payot \& Rivages, 2002.

Armengaud, Françoise. «L'animalité selon Victor Hugo : un 'alphabet formidable et profond' ». Communication au Colloque « L'animal du dix-neuvième siècle ». <http://www.equipe19.univ-paris diderot.fr/Colloque\%20animal/Page\%20titre\%20colloque.htm>

Balzac, Honoré de. «Avant-propos ». La comédie humaine. Paris : Gallimard, Bibliothèque de la Pléiade, 1976. 7-20.

Baratay, Éric. «La souffrance animale, face masquée de la protection aux dix-neuvième et vingtième siècles ». Revue québécoise de droit international (2011) : 197-216.

Baudelaire, Charles. «Le chien et le flacon». Euvres complètes I. Dir. Claude Pichois. Paris : Gallimard, Bibliothèque de la Pléiade, 1975. 284.

Buret, Eugène. De la misère des classes laborieuses en Angleterre et en France; De la nature de la misère, de son existence, de ses effets, de ses causes, et de l'insuffisance des remèdes qu'on lui a opposés jusqu'ici ; avec l'indication des moyens propres à en affranchir les sociétés. Tome 1. Paris : Paulin, 1840.

Cabanis, P.-J.-G. Rapport du physique et du moral de l'homme ( 8 éd.). Paris : Baillière, Libraire de l'Académie royale de médecine, 1844.

Duerr, Hans Peter. Nudité et pudeur. Le mythe du processus de civilisation. Tr. Véronique Bodin. Paris : Éditions de la Maison des sciences de l'homme, 1998.

Freud, Sigmund. L'inquiétante étrangeté. Paris : Gallimard, 1985.

Guichet, Jean-Luc. «La question de l'animalité, pivot du matérialisme et de la définition de l'humain chez Cabanis ». Dix-huitième siècle 42.1 (2010): 367-384. <http://www.cairn.info/revue-dix-huitieme-siecle-2010-1-page-367.htm>

Hugo, Victor. Les misérables. Tome I. Paris : Garnier, 1957. 
Lezama, Nigel. «La mise en discours du pauvre: une lumière sur le pouvoir ». Corps et représentations : une liaison dangereuse? Dir. Stéphanie Chapuis-Després, Cécile Codet et Mathieu Gonod. Paris : Harmattan, 2014. 285-295.

Marx, Karl. Le capital. Livre premier 1867, Euvres I. Paris : Éditions Gallimard, Bibliothèque de la Pléiade, 1963. 535-690.

--- et Friedrich Engels. La sainte famille ou Critique de la Critique critique contre Bruno Bauer et consorts. Tr. Erna Cogniot. Paris : Éditions sociales, 1969. <http://classiques.uqac.ca/classiques/Engels_Marx/sainte_famille/sainte famille.html >

Millet, Claude. «Souffrance animale». < http://www.equipe19.univ-parisdiderot.fr/Colloque\%20animal/Claude\%20Millet.pdf >

Serna, Pierre. «Droits d'humanité, droits d'animalité à la fin du dix-huitième siècle, ou la matrice du 'racisme social' en controverse ». Dix-huitième siècle. 42.1 (2010) : 247-263. <http://www.cairn.info/revue-dix-huitieme-siecle-2010-1-page-247.htm>

Sue, Eugène. Les mystères de Paris. Paris : Laffont, 1989.

7 À titre d'exemple, Pons-Augustin Alletz écrit Histoire des singes, et autres animaux curieux, dont l'instinct et l'industrie excitent l'admiration des hommes en 1752 ; A. Vosmaer publie sa Description d'une espèce de singe d'Amérique en 1766 ; et Petrus Camper rédige Histoire naturelle de l'orang-outang et de quelques autres singes en 1779 .

${ }^{8}$ L'anecdote apocryphe de Diderot qui rapporte que, face à l'orang-outang, le Cardinal de Polignac se serait exclamé «Parle, et je te baptise », révèle le bouleversement que le singe entame dans le statut exceptionnel que l'homme se donne aux dix-huitième et dix-neuvième siècles.

9 Je me sers de l'expression «inquiétante étrangeté » dans le sens que Freud y prête. Freud explique que « [1]e caractère d'inquiétante étrangeté ne peut en effet venir que du fait que le double est une formation qui appartient aux temps originaires dépassés de la vie psychique, qui du reste revêtait alors un sens plus aimable. Le double est devenu une image d'épouvante de la même façon que les dieux deviennent des démons après que leur religion s'est écroulée » (238-39). L'amenuisement du gouffre idéologique séparant l'animal de l'homme se manifeste, entre
} 
autres, dans la mise en discours des phénomènes de la misère et de la criminalité au dix-neuvième siècle. Fort de ce constat, il est évident que l'animal rappelle à l'homme son état pré-civilisation ; ainsi, l'animalité du criminel ou du pauvre fait surgir la peur de la dégénérescence.

${ }^{10}$ Le texte de Salaville que privilégie Serna, intitulé « De l'homme et des animaux » fut une des entrées au concours de l'Institut national de France de 1802.

${ }^{11}$ Par contre, la peinture du corps du Chourineur est marquée par la phrénologie, un autre mouvement scientifique, introduit par le médecin allemand Franz Joseph Gall, qui trouve en France un engouement pendant les premières décennies du dix-neuvième siècle.

${ }^{12}$ Bien qu'il puisse sembler cruel d'offrir une boucherie à un ancien équarisseur dont la pratique l'avait déformé, sinon traumatisé, dans la logique des Mystères de Paris, un des devoirs du prince Rodolphe est de réhabiliter les tendances inhérentes de ceux qui se sont égarés de la voie idéologique en trouvant un débouché mieux adapté. Pour récompenser un service rendu, Rodolphe a, par exemple, offert un lopin de terre en Algérie à un braconnier, Martial, et sa femme, une ancienne prostituée surnommée - pertinemment - la Louve à cause de sa nature sauvage et de toute évidence indomptable. À l'époque de la rédaction des Mystères de Paris, l'impérialisme français se voit troublé par un premier questionnement sur la décolonisation en général, et celle de l'Algérie, terre conquise depuis 1830, en particulier. Au dénouement du feuilleton, le lecteur apprend que Martial et la Louve ont prouvé leur valeur aux yeux de Rodolphe et du régime idéologique : mari et femme sont devenus tous les deux des défenseurs acharnés contre des incursions arabes dans ce territoire dominé - la Louve a même été rebaptisée «Mme Carabine » en honneur de son intrépidité. Ce qui peut sembler un cadeau empoisonné devient, dans les Mystères de Paris, une marque de la perspicacité de Rodolphe. Dans le cas du Chourineur, Rodolphe essaie de réhabiliter la nature sanguinaire inhérente de cet homme afin de réintégrer le criminel dans la société. Dans cette optique, quoi de plus convenable (et de plus jugulant) à offrir à un homme avec un penchant pour l'arme blanche qu'une boucherie ? 